\title{
Desarrollo de la dimensión afectiva de las competencias genéricas por medio del uso de la reflexión
}

\author{
MARÍA-GRACIA GONZÁLEZ NAVARRO* \\ MARGARITA ESTER MARCHANT SAN MARTÍN** \\ VÍCTOR HUGO RUÍZ RODRÍGUEZ*** \\ GRACIA NAVARRO SALDAÑA ${ }^{* * * * *}$
}

Universidad de Concepción - Chile

Recibido el 29-11-2015; primera evaluación el 03-11-2016; segunda evaluación el

08-02-2017; aceptado el 10-02-2017

\section{RESUMEN}

La dimensión afectiva de las competencias genéricas (CCGG), es fundamental para formar profesionales integrales. En el modelo educativo de la Universidad de Concepción de Chile, la dimensión afectiva se desarrolla en tercer y cuarto año de carrera. En esta investigación se presenta la experiencia de la carrera de biología. En ella se utiliza la estrategia de reflexión con trece estudiantes para desarrollar la

\footnotetext{
Asesora en competencias genéricas UDEC. Licenciada en Psicología y psicóloga por la Universidad de Concepción, MEd Psychology and Education por la Universidad de Sheffield. Contacto: mgonzalez@udec.cl

** Profesora asociada del Departamento de Zoología de la Facultad de Ciencias Naturales y Oceanográfica. Con Licenciatura de Biología Marina por la Universidad de Concepción. Magíster en Ciencias con mención en Zoología por el Departamento de Zoología, y Escuela de Graduados de la misma universidad. Doctor rer. nat., Departamento de Geología de la Universidad de Bremen, Alemania. Contacto: mmarchan@udec.cl

*** Profesor asociado, Universidad de Concepción. Licenciado en Biología. Profesor de Estado en Biología y Ciencias Naturales. Magíster en Ciencias con mención en Zoología. Contacto: vruiz@udec.cl

**** Profesora asociada del Departamento de Psicología de la Universidad de Concepción. Directora del Programa de Estudios sobre la Responsabilidad Social, directora del Programa Talentos UdeC, exdecana de la Facultad de Ciencias Sociales de la Universidad de Concepción, licenciada en Psicología por la Pontificia Universidad Católica de Chile. Magíster en Responsabilidad Social Empresarial por la Universidad de Barcelona. Doctora en Educación por la Universidad de Concepción. Contacto: gnavarro@udec.cl
} 
dimensión afectiva de las competencias genéricas. Los resultados muestran que hay un cambio en la forma en que las conciben, pasando de ser una herramienta para el buen desempeño a ser parte fundamental de la formación profesional.

Palabras clave: Competencias genéricas, dimensión afectiva, reflexión, universidad.

The affective domain of generic competencies it is essential to educate integral professionals

\section{Abstract}

In the educational model of the University of Concepcion, Chile, affective domain is developed during the third and fourth year of studies. This research presents the experience of Biology career. In it, the strategy of reflexion is used to develop the affective domain of generic competencies with 13 students. Results show that there is a change in the way that students conceive generic competencies, from being a tool for good performance to be a fundamental part of professional training.

Keywords: Generic competencies, affective domain, reflexion, higher education.

O domínio afetivo das competências genéricas é essencial para educar profissionais integrais

\section{Resumo}

A dimensão afetiva das competências genéricas (CCGG) é fundamental para formar profissionais integrantes. No modelo educacional da Universidade de Concepção no Chile, a dimensão afetiva é desenvolvida no terceiro e quarto ano de carreira. Esta pesquisa apresenta a experiência da carreira de biologia. Utiliza a estratégia de reflexão com 13 alunos para desenvolver a dimensão afetiva das competências genéricas. Os resultados mostram que há uma mudança na forma como são concebidos, passando de ser uma ferramenta para um bom desempenho, sendo uma parte fundamental do treinamento vocacional.

Palabras-chave: Competencias genéricas, dimensão afectiva, reflexion, universidad. 


\section{INTRODUCCIÓN}

Las universidades siempre han considerado en su misión el desarrollo de una formación integral de sus estudiantes. Sin duda, entre los objetivos prioritarios estaba, desde su inicio, el desarrollo social del estudiantado, aunque quizás nunca se había preocupado, hasta hoy, de medir y evaluar los logros alcanzados en cuanto a su desarrollo personal y a su contribución a una sociedad sostenible, democrática y basada en el conocimiento (Villa \& Villa, 2007).

En Europa, desde la declaración de Bolonia en 1999 (Espacio Europeo de Educación Superior - EEES, 1999), viene debatiéndose el modelo de universidad europea en el seno de las universidades y en diversos foros nacionales e internacionales se han ido analizando encuentros y cumbres ministeriales que ayudan a avanzar en el desarrollo del EEES (Praga 2001, Berlín 2003, Graz 2003 y Bergen 2005, entre otras). Sin embargo, solo recientemente, en la cumbre celebrada en Londres en el año 2007, se refiere por primera vez a la dimensión social de las universidades. En su apartado 2.18, se afirma el siguiente aspecto de la dimensión social (p. 4): La educación superior debería desempeñar un papel esencial en la promoción de la cohesión social, en la reducción de las desigualdades y en la elevación del nivel del conocimiento, destrezas y competencias en el seno de la sociedad. En Chile, el Consejo de Rectores de las Universidades Chilenas (CRUNCH), sostuvo en el año 2009, que en los planes de formación universitaria se deben generar condiciones para que los estudiantes alcancen una formación que contribuya a desarrollar plenamente sus potencialidades personales, de acuerdo con los requerimientos de la sociedad del conocimiento y que "se debe transmitir a los estudiantes, no solo la universalidad del conocimiento, sino el sentido de humanidad, — de ver al otro-, y en ello, la valorización del ser humano" (CRUNCH, 2009, p. 23).

Se puede afirmar entonces que se está reconceptualizando tanto la misión actual de las universidades como su enfoque pedagógico para dar una respuesta satisfactoria a las demandas personales, sociales y culturales de la sociedad de nuestros días. Es en este contexto que en diferentes países, incluido Chile, se ha optado por la formación en competencias genéricas (González, Maluenda \& Navarro, 2015). Las competencias genéricas contribuyen a que las personas satisfagan sus necesidades personales; consistentes con una ética e incluyendo en el concepto de éxito en la vida, las relaciones con los demás y con el entorno físico y social. Enfoque que es alternativo a considerar las competencias predominantemente desde una perspectiva de productividad y competitividad (Vilà, 2006). 
La Universidad de Concepción de Chile, consciente de las desigualdades sociales, de los cambios que trae aparejada la globalización y por consiguiente de las demandas que exige la sociedad actual, ha decidido formar transversalmente a sus estudiantes en cuatro competencias genéricas (CCGG): pensamiento crítico, comunicación, emprendimiento y trabajo en equipo interdisciplinario y, responsabilidad social. Estas están integradas al modelo educativo de la institución y se espera que todos sus egresados tengan en su desempeño profesional, las cuatro competencias que la Universidad ha considerado relevantes para responder a la sociedad. Pensando en formar un profesional de excelencia no solo en la parte disciplinar sino también en el aspecto personal y social, capaz de responder a las nuevas exigencias que la sociedad le impone dotándolo de un pensamiento crítico, de capacidad de innovar y trabajar en equipo, de comunicarse adecuadamente y de ser socialmente responsable (Navarro, Vaccarezza, González \& Catalán, 2015).

\section{Modelo UdeC de ENSEŃANZa PARA LA FORMACIÓN EN COMPETENCIAS GENÉRICAS}

A continuación se presenta una síntesis del modelo educativo de la Institución para la formación de CCGG, publicado el año 2015 (Navarro et al., 2015) en el cual las CCGG se denominan macrocompetencias genéricas, porque son de alta complejidad e incluyen un conjunto de competencias pequeñas o simples y se integran al curriculum disciplinar desde su tridimensionalidad, es decir, se considera la dimensión cognitiva, afectiva y conductual de cada una de ellas.

Este modelo considera un primer nivel (destinado a estudiantes del ciclo básico, es decir, primer y segundo año de pregrado), que introduce las CCGG en su dimensión cognitiva. Se busca que los estudiantes conozcan y comprendan las CCGG y sean capaces de identificar y analizar comportamientos profesionales asociados a ellas.

Un segundo nivel (destinado a estudiantes del ciclo licenciatura, es decir tercer y cuarto año de pregrado), pone énfasis en generar cambios afectivos en los alumnos, incluyendo desarrollo de una predisposición positiva hacia la incorporación de cada competencia en el propio comportamiento profesional, implicando a su vez una mayor autonomía en cuanto al aprendizaje de aquellas. Por otra parte, en esta etapa, el profesor acompaña y facilita este aprendizaje, a través de estrategias (e.g. supervisión de grupos pequeños). El desarrollo de este nivel supone que los resultados de aprendizaje correspondientes al primer nivel o nivel cognitivo, han sido alcanzados y por tanto los 
estudiantes manejan los aspectos correspondientes a la dimensión cognitiva de cada CCGG.

Un tercer nivel (destinado a estudiantes del ciclo profesional, es decir en práctica profesional, seminarios de investigación y proceso de titulación), implica la transferencia de los aprendizajes obtenidos en los primeros niveles a contextos profesionales. Es decir, aplicar lo aprendido durante la carrera, fuera de la universidad, poniendo en práctica contenidos, actitudes y acciones asociadas a cada CCGG.

El modelo educativo de la Universidad de Concepción (Navarro et al., 2015) sugiere diversas estrategias metodológicas para la enseñanza y evaluación de las CCGG, sin embargo, indica que con independencia de las que se decidan usar, se debe procurar cautelar los fundamentos del modelo educativo:

a) Formación y desempeño integral: se deben desarrollar las cuatro CCGG y en sus tres dimensiones.

b) Aprendizaje colaborativo: debe realizarse de forma intencional y dirigida y estar presente en la metodología del curso.

c) Evaluación auténtica: concepción de la evaluación como forma de aprendizaje, los estudiantes conocen lo que se espera de ellos y lo que se espera, corresponde a elementos que serán necesarios en su desempeño profesional.

d) Hacer explícita la enseñanza de las CCGG: en todos los niveles, desde lo declarado en documentos hasta el quehacer del profesor en la sala de clases.

Considerando lo anterior, los académicos deben procurar el uso de estrategias de enseńanza y evaluación adecuadas y adaptadas a la realidad de sus estudiantes, pero cautelando que transversalmente a ellas, estén presentes los elementos anteriores (Navarro et al., 2015).

\section{DesarRollo de LA DIMENSIÓN AFECTIVA POR MEDIO DE LA REFLEXIÓN}

Usualmente la palabra "afecto" se entiende en términos de sentimientos, emociones, motivaciones, actitudes y creencias (Boyd, Dooley \& Felton, 2006). En el caso del modelo de esta institución, la dimensión afectiva se comprende a partir de lo indicado por Goleman (1998), afectos en cuanto a que generan una disposición positiva y motivación hacia el desarrollo de una conducta. 
Por lo tanto, como se mencionó anteriormente, se espera que en la medida que los estudiantes desarrollen una predisposición positiva hacia la incorporación de cada competencia en el propio comportamiento profesional, alcancen una mayor autonomía en cuanto al aprendizaje de aquellas.

Una estrategia de enseñanza relacionada con la construcción de experiencias y conocimiento, en este caso, de tipo afectivo, es la reflexión.

Huba y Freed (2000) describen la reflexión como una actividad intelectual y afectiva en la que el individuo se involucra en explorar sus experiencias con el fin de llevarlas a nuevas apreciaciones y comprensiones. Por otra parte, de acuerdo con Boyd (et al., 2006) cuando un estudiante reflexiona sobre ideas y sentimientos, como resultado de una proceso pedagógico previamente planificado, la naturaleza del proceso de aprendizaje lo lleva a construir nuevos conocimientos y experiencias. Por lo tanto, en esa construcción sería posible revalorizar elementos ya conocidos o darles importancia a partir de una nueva forma de abordarlo.

Considerando que las CCGG son contenidos con los cuales los estudiantes han tenido algún tipo de contacto, independientemente si ha sido en su experiencia académica o no, la reflexión podría ser una estrategia adecuada para permitirles abordarlas y valorarlas en cuanto a su desempeńo profesional.

\section{EXPERIENCIA}

Este trabajo presenta una experiencia de formación de dimensión afectiva de las cuatro CCGG que se forman en la Universidad de Concepción, con estudiantes de la carrera de biología. El problema de investigación que se planteó fue ¿Cómo desarrollar la dimensión afectiva de las competencias genéricas en un curso o asignatura de anatomía animal comparada?

A partir de la revisión de la literatura especializada y de las características de la asignatura, se decidió abordar la dimensión afectiva principalmente con la estrategia de reflexión, procurando mantener presentes los cuatro pilares del Modelo Educativo UdeC para la formación en CCGG: a) formación y desempeño integral, b) aprendizaje colaborativo, c) evaluación auténtica y d) Hacer explícita la enseńanza de las CCGG (Navarro et al., 2015).

\section{TiPO DE INVESTIGACión Y VARIABLES ESTUdiadAS}

La investigación es aplicada, de tipo correlacional y considera las siguientes variables: 


\subsection{Dimensión afectiva de las CCGG}

La dimensión afectiva de las CCGG es entendida en el Modelo UdeC como el valor que los estudiantes le entregan al desarrollo de las CCGG (Navarro et al., 2015). En este estudio aquel valor se operacionaliza en términos de la importancia que los estudiantes le otorgan al desarrollo de las CCGG para el futuro desempeńo profesional y por una disposición favorable hacia trabajar para lograr aquel desarrollo.

El valor entregado a las CCGG es medido a través de la revisión de conductas declarativas, es decir del análisis de las respuestas de los estudiantes ante la pregunta ¿Qué importancia crees que tendrá el aprender sobre macrocompetencias genéricas para tu futuro desempeño profesional como biólogo?, la cual fue realizada por escrito antes (pretest) y después de la intervención (postest). Además de lo anterior, los resultados que se encontraron en la evaluación pretest fueron utilizados como diagnóstico para la planificación de la asignatura. De la misma forma, aquellos resultados de la evaluación postest, se utilizaron como retroalimentación para los académicos a cargo de la asignatura.

- Estrategia reflexiva:

La estrategia reflexiva utilizada en este curso consistió en:

Cuatro guías de reflexión individual, en las cuales los estudiantes debían ordenar sus ideas o conocimientos en relación con un tema, analizar alguna perspectiva en particular sobre ese contenido y reflexionar sobre sí mismos y su desempeño en relación a él. Cada una de esas guías se presentó por escrito y los profesores las evaluaron con una escala de valoración conocida por los estudiantes y les entregaron retroalimentación.

Cuatro guías de reflexión grupal, en las cuales los estudiantes reflexionaban en conjunto en torno a una pregunta y prepararon una pequeña exposición oral para dar a conocer al resto del curso su punto de vista.

\section{Hipótesis}

Los estudiantes que utilizan la reflexión como estrategia dirigida por los docentes en su formación, profundizan en el nivel de importancia otorgado a las CCGG. 


\section{MaTeriales Y MÉTODOS}

La intervención fue realizada por dos profesores en aula y un tercero, apoyando en la planificación de la estrategia y evaluación. Se trabajó con el universo de todos los estudiantes de tercer año de la carrera de Biología, en la asignatura Anatomía Animal Comparada, que previamente habían recibido información sobre la dimensión cognitiva de las CCGG. Este correspondió a los 14 estudiantes del curso, de los cuales, uno se retiró durante la asignatura, finalizando el curso 13 de ellos.

El trabajo se llevó a cabo durante un mes en el primer semestre del año 2015 .

\section{Resultados}

\subsection{Método de análisis}

La metodología de análisis de datos utilizada corresponde al Análisis Temático que consiste en identificar, analizar y reportar patrones en los datos (Braun \& Clarke, 2006) los cuales son considerados importantes para describir el fenómeno (Fereday \& Muir-Cochrane, 2006). Dado que la investigación es aplicada y su objetivo es reconocer una posible correlación entre la estrategia utilizada y el desarrollo de la dimensión afectiva, medida a través de la importancia otorgada a las CCGG, durante el proceso de análisis se trabajó bajo un enfoque inductivo. Lo anterior refiere a que a partir de los datos recogidos se buscó generar conclusiones y levantar información (Braun \& Clarke, 2006), que permitiera reconocer las experiencias de los estudiantes antes y después de la intervención.

\subsection{Evaluación pretest}

El análisis de las respuestas del pretest de los estudiantes, muestra que la importancia que ellos otorgan al desarrollar las CCGG, está en términos de la calidad de la respuesta que pueden entregar al medio como futuros profesionales. Los resultados revelan cuatro temas principales: a) comprensión difusa y general del campo laboral del biólogo, b) valor diferente a las CCGG, c) CCGG como respuesta a las demandas laborales y d) CCGG como respuesta al rol social del biólogo. 
a) Comprensión difusa y general del campo laboral del biólogo

De acuerdo a los datos, todos los estudiantes relacionan la importancia de desarrollar CCGG con su futuro rol profesional. Sin embargo, sus respuestas muestran un conocimiento general y superficial de las áreas de desempeño de los biólogos, en el cual resalta principalmente la investigación:

"Por nuestra disciplina, probablemente sea necesario efectuarlo con más gente, de distintas disciplinas, para ayudarnos de que sea lo más completo posible" (2P9-2P10).

"Creo que será importante tener conocimiento sobre las competencias debido a que los biólogos generalmente trabajan en equipo, ya sea en empresas o proyectos o trabajos de investigación, por lo tanto, debe conocer la importancia del trabajo interdisciplinario para mantener buenas relaciones sociales y disciplinarias" (10P44-10P46).

"Saber trabajar en equipo, lo cual se necesita en nuestra área para realización de investigación y salidas a terrenos" (11P50 -11P51).

"El aprender sobre competencias genéricas, desde la mirada del biólogo, consistirá en un importante pilar a la hora de afrontar desafíos donde pongan a prueba capacidades como el trabajo en equipo, lo cual es muy frecuente, ya que la mayoría de las investigaciones se realizar con colaboraciones de especialistas en áreas determinadas" (12P53-12P55).

La comprensión de este tema es fundamental porque se relaciona con el nivel de profundidad de las reflexiones que los estudiantes pueden tener sobre su rol profesional (UdeC, 2016). En la medida en que no está en sus esquemas conceptuales la diversidad de áreas disciplinares en las que se pueden desempeñar y en lo que consiste cada una de ellas, se dificulta que puedan ver la aplicación que tendrán las CCGG en ellas y por lo tanto, otorgarles importancia para su futuro profesional.

De lo anterior se infiere que el desarrollo de la dimensión afectiva de las CCGG se podría ver dificultado por el desconocimiento de las áreas de desempeño profesional. $\mathrm{Al}$ no conocer las futuras necesidades profesionales se limita la identificación y valoración de la aplicación de las CCGG en el futuro rol profesional.

b) Valor diferenciado a las CCGG

Del análisis de los datos se concluye que al momento de comenzar el curso los estudiantes no valoraban el desarrollo de las cuatro CCGG (pensamiento crítico, comunicación, emprendimiento y trabajo en equipo, y responsabilidad social) de la misma forma, existiendo claras preferencias por unas que por otras. 
De esto, se puede inferir que su percepción de un desempeño profesional adecuado no integra las cuatro CCGG, sino que solo requiere de algunas para ser el esperado.

En el caso de la competencia de trabajo en equipo, el 71,43\% de los estudiantes de la asignatura Anatomía Animal Comparada valoran su desarrollo para el desempeño profesional, mencionándola explícitamente y siendo ella a la que más se refieren. No indican cuál sería la forma adecuada de trabajar en equipo, sin embargo, sí relacionan esta competencia con la necesidad de trabajar junto a profesionales de otras disciplinas.

"El poder relacionarnos mejor con nuestro entorno laboral, de forma de poder ser tanto un buen compañero, como de ser un aporte solido a la hora de realizar un trabajo, que, por nuestra disciplina, probablemente sea necesario efectuarlo con más gente, de distintas disciplinas, para ayudarnos de que sea lo más completo posible" (2P8 - 2P10).

"El aprender sobre competencias genéricas, desde la mirada del biólogo, consistirá en un importante pilar a la hora de afrontar desafíos donde pongan a prueba capacidades como el trabajo en equipo, lo cual es muy frecuente, ya que la mayoría de las investigaciones se realizar con colaboraciones de especialistas en áreas determinadas" (12P53 - 12P55)

El 35,71\% de los estudiantes valoran explícitamente el desarrollo de la CCGG pensamiento crítico, sin especificar la forma en la cual actuaría un biólogo con pensamiento crítico.

"Nos permitirá tener un pensamiento crítico... una vez que hayamos ejercido como profesionales" (8P37-8P38).

"Desempeñarnos en el ámbito laboral... aplicando un pensamiento crítico" (9P42-9P43)

Solo un participante $(7,14 \%)$, sí hace una relación entre las características del pensamiento crítico con la capacidad de responder a los desafíos de la disciplina.

"También el pensamiento crítico, será fundamental a la hora de crear una postura con respectos a decisiones que comprometan un análisis ético con relación a un tema en particular" (12P44 - 12P57).

El 28,57\% de los estudiantes menciona explícitamente la CCGG responsabilidad social como importante para desarrollar en el futuro desempeńo profesional. Sin embargo, no realizan mayor análisis sobre los beneficios o ventajas que les brinda.

"El saber sobre competencias genéricas es importante o más bien será importante en nuestra vida profesional, ya que nos dará herramientas extras que muchas veces nos enseñan como parte de la malla curricular. Estas 
herramientas tienen como objetivo tener en cuenta varios aspectos como la responsabilidad social..." (6P23 -6P26).

Solo uno de los participantes describe la manera en que la competencia podría afectar de forma positiva su desempeño profesional, haciendo énfasis en los impactos que su comportamiento tendría sobre la sociedad:

"La importancia del conocimiento y aplicación de las competencias se relaciona con la formación de profesionales integrales y con conciencia social. Que comprendan el contexto y la importancia de su trabajo y el efecto que este tiene en el medio. Comprender también la importancia de la existencia del trabajo biológico y las consecuencias que este puede traer con su aplicación deficiente o nula" (5P18-5P21).

La CCGG emprendimiento y trabajo en equipo interdisciplinario también fue valorada explícitamente por un $28,57 \%$ de los estudiantes. Las respuestas de los estudiantes no muestran profundización en sus respuestas, solo mencionando la competencia como importante para el futuro:

"Nos permitirá tener un pensamiento crítico, guiarnos en el emprendimiento, ser buenos líderes una vez que hayamos ejercido como profesionales, etc." (8P37 - 8P38).

En el caso de esta competencia, un estudiante $(7,14 \%)$ la relaciona con la capacidad de los biólogos para responder con éxito a los avances científicos:

"Además como biólogos es importante el emprendimiento para crear nuevos proyectos de investigación debido a que la ciencia siempre avanza y abre nuevas puertas de conocimiento" (10P46-10P48).

Por último, la CCGG comunicación solo fue considerada importante explícitamente por dos estudiantes (14,29\%). De ellos, uno la valoró en términos de los beneficios que puede traer al trabajo en equipo y en el segundo caso, en relación a la posibilidad de difundir mejor la ciencia.

El identificar que los estudiantes valoran de forma desigual a cada una de las CCGG, es un elemento importante de tener en cuenta al momento de planificar una asignatura para el desarrollo de las competencias. Uno de los pilares del modelo educativo de la Universidad, es la formación integral, es decir, que los estudiantes desarrollen las tres dimensiones de las competencias, pero además que desarrollen las cuatro de forma similar (Navarro, Vaccarezza, González \& Catalán, 2015) . Por lo tanto, se hace necesaria la puesta en práctica de estrategias específicas para lograr este aspecto.

c) CCGG como respuesta a las demandas laborales

Un tema relevante que se desprende del análisis de los datos, es la percepción que tienen los estudiantes de las CCGG como una herramienta para responder 
adecuadamente a las demandas laborales, así lo menciona un 64,29\% de ellos. Esas respuestas están en términos de: 1) formarse como profesionales integrales y de excelencia en términos disciplinares y personales, lo cual es requerido en el mercado laboral actual.

"Yo creo que la importancia radica en aprender qué se nos exige en el ambiente laboral para surgir, y aprenderlas desde ya para que todos tengamos las mismas posibilidades de encontrar trabajo" (7P29-7P30).

"La importancia la cual nos peoría otorgar las competencias genéricas sería el desarrollarnos integralmente como profesionales. Saber trabajar en equipo, lo cual se necesita en nuestra área para realización de investigación y salidas a terrenos. El poder comunicarnos bien nos ayuda a realizar efectivos estudios para la ciencia y así aportar con ella. Nos permitirá el poder de emprendimiento para ser constante en nuestro trabajo y desempeño" (11P49-11P52).

El valor que los estudiantes le otorgan a las CCGG como una herramienta para aumentar la empleabilidad, está altamente relacionado con lo mencionado tradicionalmente en la literatura internacional, donde uno de los principales elementos para su desarrollo está en términos de lo solicitado en el mercado laboral (Bennett, Dunne \& Carré, 2000; Hager, Holland \& Beckett, 2002; Centre for Developing and Evaluating Lifelong Learning 2007).

La segunda forma en la que los estudiantes conciben las CCGG como una herramienta para responder adecuadamente a las demandas laborales, es a través de que su desarrollo les permitirá 2) enfrentar con éxito los desafíos propios de la Biología, en cuanto a los avances científicos que surjan y su capacidad de adaptarse a ellos:

"En la biología la responsabilidad, la crítica y la resolución de problemas permite formar un profesional más capaz de mira a la evolución que experimenta la ciencias naturales" (4P16 - 4P17).

"El aprender sobre competencias genéricas, desde la mirada del biólogo, consistirá en un importante pilar a la hora de afrontar desafíos donde pongan a prueba capacidades como el trabajo en equipo, lo cual es muy frecuente, ya que la mayoría de las investigaciones se realizar con colaboraciones de especialistas en áreas determinadas. También el pensamiento crítico, será fundamental a la hora de crear una postura con respectos a decisiones que comprometan un análisis ético con relación a un tema en particular" (12P53-12P57).

Uno de los resultados de aprendizaje esperados del trabajo de la dimensión afectiva, es que los estudiantes valoren la importancia de las CCGG para su futuro desempeño profesional. El enfrentar adecuadamente desafíos profesionales, es parte de ese desempeńo, por lo que se puede inferir que el estado de los estudiantes antes de comenzar la intervención, ya era de una dispo- 
sición afectiva que permitía recibir positivamente la temática y estrategia de enseñanza. Krathwohl, Bloom y Masia (1964) categorizan a las personas que tienen una disposición a prestar atención a un estímulo en el primer nivel de su taxonomía del domino afectivo: recibir.

d) CCGG como respuesta al rol social del biólogo

Un importante tema que aparece en las reflexiones de los estudiantes sobre el valor de las CCGG es que las consideran herramientas que les permitirán responder mejor ante la sociedad desde su profesión.

"La importancia del conocimiento y aplicación de las competencias se relaciona con la formación de profesionales integrales y con conciencia social. Que comprendan el contexto y la importancia de su trabajo y el efecto que este tiene en el medio. Comprender también la importancia de la existencia del trabajo biológico y las consecuencias que este puede traer con su aplicación deficiente o nula. El tema de las competencias genéricas debe tener una importancia y un planteamiento relacionado con la colectividad y con las relaciones y temáticas sociales $(5 \mathrm{P} 18-5 \mathrm{P} 22)$.

"Teniendo en cuenta que la sociedad en la cual vivimos actualmente requiere que los profesionales conozcan estas herramientas, ya que gracias a estas podrían mejorar la calidad de vida que hoy en día llevamos como seres humanos" (6P26-6P28).

Esta idea se relaciona con el rol social de las universidades: formar profesionales que contribuyan al desarrollo social; el cual también es parte de la misión y visión de la Universidad en la que se lleva a cabo esta experiencia "el propósito de contribuir efectivamente al desarrollo humano, económico y social sustentable de la región y el país" (Universidad de Concepción, 2011). Este tema es mencionado solo por el 14,29\% de las respuestas durante el pretest, sin embargo, al complementarlo con el tema anterior "competencias genéricas como respuesta a las demandas laborales", se potencian facilitando la intervención de los académicos con el curso.

\subsection{Evaluación postest}

El análisis de las respuestas de los estudiantes en el postest, muestra que la importancia que ellos otorgan a desarrollar CCGG está en términos de la calidad de profesional que serán. Es decir, el desarrollo de CCGG es un beneficio personal que implica ser mejores profesionales y, por lo tanto, desempeñarse de buena forma. Esta es una diferencia importante con los resultados encontrados en el pretest, en el cual la importancia estaba en términos de responder positivamente 
a las exigencias del medio. Antes de la intervención, el foco de la importancia estaba puesto en el exterior, en responder a lo que se les solicitaba. En cambio, después de la intervención, el foco está en el interior, en el desarrollo personal de cada estudiante. Los temas identificados se describen a continuación.

a) Comprensión concreta y específica del campo laboral del biólogo

De acuerdo al análisis de los datos, durante la intervención los estudiantes adquirieron una concepción más profunda de las áreas de su futuro desempeño profesional. Al describir las formas en las cuales las CCGG traen beneficios, todos los participantes, se refieren a diferentes aspectos de su campo laboral y de forma específica con relación a cómo lo hacían al inicio de la asignatura. Esto sugiere un aprendizaje de las futuras áreas de desempeño laboral, el cual se podría relacionar con las temáticas de reflexión de la intervención, las que tuvieron relación con este aspecto.

"El aprender y por supuesto aplicar estas macrocompetencias tiene una importancia efectiva en ese campo de la investigación (biología) más en lo que pretendo que es la investigación fundamental, donde cada aspecto de las citadas cuatro MCG son de uso en esta ciencia de la vida donde se estructuran ideas con ayuda de otros profesionales competentes cada uno en su área (trabajo interdisciplinario) aunando esfuerzos y presentando este mismo trabajo a la comunidad científica, lenguaje formal, o a la ciudadanía, lenguaje informal, teniendo en cuenta su aplicación inmediata o posterior quizás en los intereses de la sociedad (R. Social)" (15P8-15P13).

"Creo que aprender sobre macrocompetencias genéricas será fundamental en mi desempeño profesional biólogo, principalmente porque el pensamiento crítico es en esencia la labora del ideal científico, quien debe estar analizando la nueva información que incesantemente llega desde el medio, determinando su veracidad e integrándola con su perspectiva personal cuando corresponde." (16P23-16P26).

b) Valor a la formación integral de las cuatro CCGG

Un importante aspecto que aparece después de la intervención es que los estudiantes atribuyen valor a las cuatro CCGG que se enseñan en la Institución y no a algunas por sobre otras. Es decir, consideran que para desempeñarse adecuadamente, su comportamiento debe contar con las cuatro. En este aspecto hay una diferencia importante entre el pre y el postest. En la primera instancia, los estudiantes mencionaron principalmente algunas competencias, mientras que en la segunda oportunidad, el $100 \%$ de ellos se refirieron a las cuatro, entregándoles una importancia similar. 
“Creo que las competencias tendrán una fuente importancia en mi desempeño profesional, ya que cada una de ellas presenta un importante valor a la hora de ejercer la profesión, como por ejemplo, en la investigación será muy útil el uso del pensamiento crítico, pudiendo analizar de varias maneras diferentes un problema para llegar a una mejor respuesta. Otra buena ocasión de aplicar las competencias sería al momento de trabajar en equipo, ya que como hemos comentado anteriormente, en nuestra vida profesional como biólogos tendremos muchas instancias en las que será necesario trabajar en un equipo interdisciplinario, siendo el caso, debemos ser un aporte eficiente al grupo dándoles confianza en nuestros conocimientos y tener la capacidad de motivarlos a seguir adelante con el trabajo. Tanto a la hora de exponer nuestro trabajo como en el día a día, será necesaria la comunicación, que debe ser clara, coherente y adecuada al público en contexto, cosa que los receptores queden con la misma idea en la cabeza que el emisor, sin haber malentendidos en la información. Y por último, pero no menos importante, la responsabilidad social, ya que no trabajamos porque sí, sino por el bien de una sociedad a la que debemos retribuirle de alguna forma, ya sea mediante conocimientos o como enseñanzas, pero lo mejor sería realizar un trabajo a conciencia, siendo que nuestro trabajo podría tener un directo impacto en el ambiente. Además, muy probablemente los resultados de nuestra investigación serán usados tanto por los profesionales biólogos como de otras disciplinas, así que debemos asegurarnos de hacer el mejor trabajo posible, sin dejar cabos sueltos ni información completa" (19P66 - 19P80).

$\mathrm{Al}$ analizar los datos, se destaca que cada una de las competencias fue relacionada principalmente con un área de desempeño laboral. De esta forma, el pensamiento crítico fue relacionado principalmente con la investigación y la generación de conocimiento, a la cual los participantes consideran como la labor esencial del biólogo. El emprendimiento y trabajo en equipo, fue relacionado con la capacidad de generar respuestas más completas a los desafíos de la Ciencia, frente a los cuales muchas veces no pueden responder desde solo un área de experticia. La competencia comunicación la relacionan principalmente con la divulgación científica y con la capacidad de expresarse de una forma que su interlocutor comprenda, lo cual consideran como su responsabilidad. Finalmente, la responsabilidad social, la relacionan con la capacidad de darse cuenta de los impactos que podrían tener sus acciones para la sociedad y, por lo tanto, procurar que sean positivas.

Este resultado es coherente con los objetivos esperados del curso y del modelo educativo: valorar las cuatro CCGG para el futuro desempeño profesional. 
c) CCGG como fundamento del desempeño profesional

El análisis de los datos muestra que para los estudiantes de biología las CCGG son necesarias para su desempeño profesional, integrándolas a las competencias propias de la disciplina. Es decir, consideran que en la medida que logren desarrollarlas, podrán desempeńarse adecuadamente. Esto es diferente a lo encontrado en el pretest, en el cual los estudiantes las consideraron como un valor agregado a su desempeño.

Como se antindicó anteriormente, el 100\% de los estudiantes mencionan las cuatro competencias genéricas como importantes a desarrollar y las relacionan con algún área de su desempeño. Es importante destacar que en el postest aparecen dos áreas de desempeńo que no se consideraron en el pretest: divulgación científica y responsabilidad social. Esta última fue mencionada en el pretest por dos estudiantes como parte del rol social que tiene el biólogo, mientras que en el postest fue mencionada por todos los estudiantes, como inherente al desempeño profesional.

Si las respuestas de los estudiantes se consideran como sus creencias y se intenta categorizarlas a partir de ellas en un nivel de los propuestos por Krathwohl, Bloom y Masia (1964) en su taxonomía del dominio afectivo, se identifica un cambio a partir de lo considerado en el pretest. Como se mencionó previamente, antes de la intervención los estudiantes se ubican en el nivel de "recepción", mientras que después de aquella, están en el nivel de "valoración". Es decir, los estudiantes aceptan y prefieren un valor, en este caso, la formación integral como fundamento del desempeño profesional y se muestran comprometidos con su desarrollo.

d) Actitud positiva hacia el trabajo para desarrollar CCGG

Además de una valoración explícita hacia las CCGG, el análisis de los datos permite inferir que se ha desarrollado una disposición positiva hacia el trabajo requerido para desarrollar las competencias. El 23\% de los estudiantes indica explícitamente que el conocimiento no es suficiente para desarrollar e incorporar al propio comportamiento las CCGG, manifestando su interés por continuar realizando acciones para lograr su desarrollo. El modelo de formación de la Universidad en la que se lleva a cabo esta experiencia, está integrado a las mallas curriculares y considera la tridimensionalidad de las CCGG para su formación, es decir, su composición por una dimensión cognitiva, afectiva y conductual y lo largo de la carrera, se va haciendo énfasis en una de aquellas. Por lo tanto, la percepción de los estudiantes de estar trabajando solo a nivel conceptual y su necesidad de profundizar en lo conductual, es precisamente 
un resultado esperado de la dimensión en la que ellos están haciendo énfasis en este momento, dimensión afectiva.

"Aun así, el APRENDER sobre las competencias no es la clave... Se puede saber en qué consisten las competencias genéricas y cuáles son los puntos más importantes a enfatizar. Sin embargo, la ejecución es donde es importante, y por ende, una persona que quiera ser buena eficiente en su uso debe hacerlo constantemente. Es necesario ejercer constante y conscientemente las competencias genéricas, hacer el ejercicio mental de activamente ser presentado ante una situación y abordar esta con las cuatro competencias. Así, en el momento en que como profesionales nos enfrentemos a cualquier situación, tengamos, por así decirlo, el entrenamiento, de cómo afrontar una situación con una buena comunicación, poder realizar un buen trabajo en equipo, pensar críticamente al tomar las decisiones, y que estas sean las mejores para con la sociedad gracias a la responsabilidad social” (25P182-25P190).

A partir de los resultados obtenidos del análisis de los datos, se realizó una comparación para identificar los cambios más significativos y relacionarlos con la intervención realizada, en la Tabla 1 se ilustra el cuadro comparativo.

\section{Tabla 1. Resumen de análisis de resultados de evaluación pretest y postest}

\begin{tabular}{|c|c|c|}
\hline Categorias de comparación & Evaluación Pretest & Evaluación Postest \\
\hline $\begin{array}{l}\text { Concepción de competencias } \\
\text { genéricas }\end{array}$ & $\begin{array}{l}\text { Herramientas que entregan un } \\
\text { valor agregado a la formación } \\
\text { profesional. }\end{array}$ & $\begin{array}{l}\text { Capacidades esenciales para } \\
\text { desempañarse como profesional. }\end{array}$ \\
\hline $\begin{array}{l}\text { Valoración de las competen- } \\
\text { cias genéricas }\end{array}$ & $\begin{array}{l}\text { Se consideran importantes de } \\
\text { desarrollar para el futuro des- } \\
\text { empeño profesional. }\end{array}$ & $\begin{array}{l}\text { Se consideran esenciales en la } \\
\text { formación profesional. }\end{array}$ \\
\hline $\begin{array}{l}\text { Valoración de las cuatro } \\
\text { competencias }\end{array}$ & $\begin{array}{l}\text { Valoración diferenciada a } \\
\text { las competencias genéricas. } \\
\text { Se valora principalmente el } \\
\text { trabajo en equipo. }\end{array}$ & $\begin{array}{l}\text { Valoración similar de las cuatro } \\
\text { competencias. Se da importancia } \\
\text { a la formación que las integra, }\end{array}$ \\
\hline $\begin{array}{l}\text { Objetivo de formación en } \\
\text { competencias genéricas }\end{array}$ & $\begin{array}{l}\text { Responder adecuadamente a las } \\
\text { demandas laborales y sociales. }\end{array}$ & Convertirse en profesional. \\
\hline $\begin{array}{l}\text { Conocimiento y compren- } \\
\text { sión del rol profesional }\end{array}$ & Difusa y general. & Amplia y específica. \\
\hline Actitud hacia el trabajo & No se observa. & $\begin{array}{l}\text { Disposición positiva a trabajar } \\
\text { para desarrollar las competencias. }\end{array}$ \\
\hline $\begin{array}{l}\text { Nivel de taxonomía } \\
\text { de dominio afectivo } \\
\text { (Krathwohl et al., 1964) }\end{array}$ & Recepción & Valoración \\
\hline
\end{tabular}




\section{Discusión}

El desarrollo de la dimensión afectiva de las CCGG es una parte esencial para lograr egresar profesionales íntegros. En la medida que los estudiantes le den importancia a formarse en estos aspectos y se preocupen de hacerlo hay más posibilidades de que efectivamente lo hagan y lleguen a desempeñarlas en su comportamiento profesional.

En el caso de esta experiencia, dadas las características del curso, se decidió trabajar con la estrategia de reflexión, por medio del uso de guías de trabajo individuales y grupales.

Para evaluar el efecto de la estrategia utilizada en el curso se aplicó una evaluación pre y postest, lo que permitió reconocer la importancia que los estudiantes atribuyen al desarrollo de CCGG.

El análisis de los resultados, mostró importantes cambios cualitativos, los cuales tienen que ver principalmente con la forma en que los estudiantes conciben el rol profesional. Antes de la intervención, ellos lo pensaban como un rol primordialmente técnico para el cual el desempeño de excelencia tenía que ver con aspectos disciplinares. En este caso, las CCGG le entregaban un valor agregado a su desempeńo, aumentando sus posibilidades en el mercado laboral. Luego de la intervención, los estudiantes incorporaron las CCGG a su desempeño profesional, como parte fundamental del comportamiento. Por lo tanto, el ser profesional supone el desarrollar las cuatro CCGG. Este cambio conceptual es importante e implica una valoración diferente a las competencias. Mientras que en el primer caso se ven como herramientas de mucho valor que se suman a su formación, por lo tanto pueden ser profesionales aun sin haberlas desarrollado, en el segundo caso, al pasar a ser parte de lo que requieren para ser profesionales, deberán de tomar acciones para desarrollarlas.

Un elemento importante que puede haber promovido el efecto positivo que tuvo el uso de la estrategia, es que los estudiantes poseían conocimientos previos de las CCGG, por lo tanto, al reflexionar al respecto tuvieron una base para organizar sus ideas y experiencias. De la misma forma, un elemento que pudo haber dificultado el logro, pero que una vez detectado se decidió considerar en la asignatura para que no lo hiciera, fue el desconocimiento inicial de los estudiantes en relación al rol profesional. En la medida en que no tenían información precisa sobre las áreas en las que se podrían desenvolver, se dificultaba el que pudieran pensar en los beneficios o amenazas que podría implicar el desarrollar o no de las competencias. Por lo tanto, se sugiere controlar este aspecto al trabajar con la dimensión afectiva.

El análisis de los resultados, también mostró una diferencia importante en la forma de las respuestas. En el pretest, eran cortas, generales y sin análisis 
crítico. Mientras que en la evaluación de postest las respuestas fueron extensas, específicas y críticas. Esto se puede relacionar con un avance en la dimensión afectiva de los estudiantes, ya que en la medida que ellos se interesan en estos contenidos, se involucran en la discusión. Pero también se podría relacionar, con el mes de exposición a actividades reflexivas, por lo que su capacidad de reflexión puede también haber mejorado por medio de la práctica. Independientemente de las razones, los estudiantes mostraron una mejora importante en su capacidad de expresar ideas, por lo que se sugiere seguir promoviendo actividades de este tipo con ellos.

Por último, la estrategia utilizada mostró importantes resultados en cuanto al desarrollo de la dimensión (revisar Tabla 1), sin embargo, es importante precisar que para que tenga efectos positivos, es necesario que sea parte de una situación planificada. Es decir, tener pleno conocimiento de los resultados de aprendizajes esperados y desarrollar actividades que permitan lograrlos. Es necesario, preparar preguntas y actividades de cierre con los estudiantes que les permitan terminar de completar sus nuevos esquemas de conocimientos y darles los espacios y tiempos necesarios para eso. Trabajar con actividades reflexivas, generalmente requiere de mayor tiempo en el aula, los estudiantes tienden a involucrarse activamente y, por lo tanto, a extenderse en sus respuestas cuando se les da espacio a comentar. Es importante, considerar eso en la planificación y también lo es, permitir que se generen esas discusiones. De esa forma se facilita su aprendizaje significativo por medio de esta estrategia.

\section{Conclusión}

El uso de la reflexión para el desarrollo de la dimensión afectiva de las CCGG parece ser adecuado para alcanzar los resultados de aprendizaje esperados en estudiantes de tercer ańo de la carrera de biología. Es decir, para que ellos valoren las cuatro CCGG como importantes para su desempeño profesional y que desarrollen una actitud positiva hacia el trabajo para desarrollarlas.

\section{REFERENCIAS BIBLIOGRÁFICAS}

Bennett, N., Dunne, E. \& Carré, C. (2000). Skills development in higher education and employment. Buckingham: SRHE.

Boyd, B., Dooley, K. \& Felton, S. (2006). Measuring learning in the affective domain using reflective writing about a virtual international agriculture experience. Journal of Agricultural Education, 47(3), 24-32. https://doi. org/10.5032/jae.2006.03024 
Braun, V. \& Clarke, V. (2006). Using thematic analysis in psychology. Qualitative Research in Psychology, 3, 77-101. https://doi.org/10.1191/ 1478088706qp063oa

Centre for Developing and Evaluating Lifelong Learning (2007). The teaching, learning and assessment of generic employability skills. Recuperado de http:// www.swslim.org.uk: http://www.swslim.org.uk/research/ges/index.asp

Consejo de Rectores de las Universidades Chilenas. (2009). El Consejo de Rectores ante los desafios de las Universidades Chilenas en el Bicentenario. Santiago de Chile: CRUNCH.

Espacio Europeo de Educación Superior (1999). Declaración de Bolonia. Recuperado de http://eees.umh.es/contenidos/Documentos/DeclaracionBolonia.pdf

Fereday, J. \& Muir-Cochrane, E. (2006). Demonstrating rigor using thematic analysis: a hybrid approach of inductive and deductive coding and theme development. International Journal of Qualitative Methods, 5(1), 1-11. https://doi.org/10.1177/160940690600500107

Goleman, D. (1998). Working with Emotional Intelligence. Nueva York: Bantam Books.

González Navarro, M., Maluenda Albornoz, J. \& Navarro Saldaña, G. (2015). Experiencias de la formación de competencias genéricas en la educación superior: casos basados en el conocimiento construido en la Universidad de Concepción, Chile. G. Navarro (ed.). Concepción, Chile: Universidad de Concepción.

Hager, P., Holland, S. \& Beckett, D. (2002). Enhancing the learning and employability of graduates: the role of generic skills. Business/Higher Education Round Table, 9, 3-16.

Huba, M. \& Freed, J. (2000). Learner-centered assessment on college campuses: Shifting the focus from teaching to learning. Boston: Allyn and Bacon.

Krathwohl, D., Bloom, B. \& Masia, B. (1964). Taxonomy of educational objectives. The classification of educational goals. Handbook II: Affective domain. Nueva York: David McKay Co.

Navarro, G., Vaccarezza, G., González, M-G. \& Catalán, R. (2015). Construcción de conocimiento en educación superior: Educación de competencias genéricas en la Universidad de Concepción (Chile). G. Navarro (ed.). Concepción, Chile: Universidad de Concepción.

Universidad de Concepción (2016). Planes y Programas de Estudio de carreras de la Facultad de Ciencias Naturales y Oceanográficas. Carrera Biología. Universidad de Concepción, Documento interno de trabajo. Síntesis publicada en http://admision.udec.cl/?q=node/29.

Vilà, R. (2006). La dimensión afectiva de la competencia comunicativa intercultural en la educación secundaria obligatoria: escala de sensibilidad intercultural. Revista de Investigación Educativa, 24(2), 353-372.

Villa, A. \& Villa, O. (2007). El aprendizaje basado en competencias y el desarrollo de la dimensión social en las universidades. Educar, 40, 15-48. 